\title{
Concerning Pure and Applied Phenomenology: A Response to Misgeld ${ }^{1}$
}

\section{Amedeo Giorgi}

Duquesne University

In a recent issue of this journal, Misgeld (1983) reviewed Luckmann's (1983) article on common sense and science and endorsed the view apparently held by Luckmann that "an appropriate clarification of the relation between common sense and science cannot be achieved by exclusively adopting the orientation of phenomenological research" (Misgeld, p. 195). Misgeld praises Luckmann for introducing the perspective of the sociology of knowledge because he feels that an historical vantage point is thereby introduced whereby one "can identify the changing relation between common sense and science" (p. 195). This is an advantage for Misgeld because questions can then be raised about "the place of common sense knowledge and scientific knowledge in contemporary society which phenomenological inquiry as such does not convey" (p. 195). Misgeld then goes on to criticize my comments on Luckmann because they are committed to a Husserlian interpretation of phenomenology.

The issues that Misgeld's article raises that have to be addressed in order to understand my article properly are (1) the context of my remarks, (2) the a priori bias that seems to be present in his contrasting of the positions, and (3) the question of whether he does justice to Husserl's views. Let us take up these issues one at a time.

\section{Context and Bias}

We will treat the first two issues together because they can be dealt with rather quickly. Misgeld shows little appreciation of the fact that my article was a commentary on Luckmann and thus entirely responsive in terms of themes, perspectives and arguments rather than initiary. Moreover, I chose to respond to the relationship between common sense and science in terms of my own discipline of psychology since I was aware of Titchener's and Grace Adams' comments on the issue. Even so, in my comments I said that I basically agree with Luckmann's statements, which would include taking account of sociology of knowledge factors, but only suggested certain criticisms about the assumptions and implications of his analyses. My major critique was that Luckmann did not mention the "science of the lifeworld" that Husserl (1970b) was suggesting to help understand the relationship between common sense and science, especially since Luckmann himself wrote: "I shall first consider common 
sense and science in phenomenological perspective in order to see how they originate in human consciousness" (p. 59). My only point here is to make explicit the secondary role my comments play because of the function of the context of the original situation in which the lectures were given. Had I to write on the issue of common sense, science and the lifeworld, I would have approached the topic very differently and certainly more comprehensively than I was able to as a discussant.

Secondly, it should be pointed out that in his very presentation of Husserlian phenomenology as opposed to other interpretations of the movement, Misgeld's rhetoric reveals an a priori bias that seriously raises the question of fairness to Husserl. The adjectives assigned to Husserlian phenomenology are pejorative, those for the type Misgeld wants, relevant and praiseworthy. Thus, Misgeld says that "phenomenology in its purest form does not provide readings (Taylor, 1971) of our cultural situation" (p. 195); he also writes that Husserl and I call for "the renewal of the orthodox programme in phenomenology" (p. 197), and that this "orthodox insistence on interminable description in phenomenology" (p. 197) cannot deal with the presence of a "technocratic ideology"; and later he states that "one can no longer remain exclusively committed to merely descriptive phenomenology" (p. 197) and finally, Husserl's project is described as "the elusive programme of a phenomenological 'science' (universal knowledge)" (p. 199) (all emphases mine).

On the other hand, what Misgeld endorses as a "hermeneutically reformulated phenomenological orientation" is "aware of its own limits" (p. 199); it is a phenomenology that "aims at the formation of critical and interpretive competence in the practice of reflection" (p. 198); his view would involve a "process of deliberation" which would intend the "resolution of cultural conflicts by aiming at broader and more encompassing understandings" (p. 198); and it would "instill a respect for the 'phenomena" " (p. 199) (all italics are mine).

Thus, we have words like "interminable," "merely," "elusive," for Husserl and "critical and interpretive," "more encompassing," and "respect" for Misgeld. Hence, the very features that have to be proven are stated at the outset and are used as criteria for discriminating a "good" and "bad" phenomenology. My claim is that all of the terms that Misgeld uses for his version of phenomenology can be used for "orthodox Husserlian phenomenology." More important, however, is the question of the accuracy of Misgeld's interpretation of Husserl. My claim is that he detracts from the Husserlian project and makes a "straw man" out of his position. By contrast, his position looks stronger. Let me indicate how this is so. 


\section{Husserl on Phenomenology vs. Misgeld's Interpretation}

It seems to me that there are five major critiques that Misgeld makes of Husserl and we shall consider each one in turn.

\section{(1) Phenomenology and Cultural Readings}

We have already noted that Misgeld claimed that phenomenology in its purest form could not provide cultural readings. He bases his argument on Taylor (1971) but Taylor does not assume a phenomenological perspective in that article, nor does he cite Husserl. However, that Husserl himself did not believe that he could not do cultural analyses is evident from many passages, but the following quotes from the "Origin of Geometry" in the Crisis should be sufficiently convincing:

rather, to understand geometry or any given cultural fact is to be conscious of its historicity, albeit "implicitly." This, however, is not an empty claim; for quite generally it is true for every fact given under the heading of "culture," whether it is a matter of the lowliest culture of necessities or the highest culture (science, state, church, economic organization, etc.) that every straight forward understanding of it as an experiential fact involves the "co-consciousness" that it is something constructed through human activity.

This is of course also to say that the whole of the cultural present, understood as a totality, "implies" the whole of the cultural past in an undetermined but structurally determined generality. To put it more precisely, it implies a continuity of pasts which imply one another, each in itself being a past cultural present. And this whole continuity is a unity of traditionalization up to the present, which is our present as (a process of) traditionalizing itself in a flowing-static vitality. This is ... an undetermined generality, but it has in principle a structure which can be much more widely explicated by proceeding from these indications, a structure which also grounds, "implies," the possibilities for every search for and determination of concrete, factual states of affairs. (Husserl, 1970b, pp. 370-371)

Thus, not only does Husserl believe he can "read" culture, but also ground research into culture methodically and systematically. Misgeld wants to examine "selectively determined situations" (p. 198), those already "in conflict." Husserl's approach already allows for that goal as the last sentence of the second paragraph makes clear. However, to analyze them fully and most relevantly, one would have to uncover the structure that grounds those states of affairs, which includes the "inner historicities of the persons taking part" (Husserl, 1965, pp. 371-372) in the superficial structure within the social-historical world. But seeking the subjective grounds of the phenomena does not exclude taking into account the socialhistorical meanings as such. This leads to the next point. 
When Luckmann (p. 59) speaks of common sense and science, he allows that they both can be subjective and social. When I spoke of meanings (and Misgeld quotes me correctly on p. 196) I allowed for the possibility that they could be subjective or intersubjective. However, when Misgeld speaks of Husserl's project, he only allows for the analysis of "subjective meaning." Thus, just after quoting me where I speak of "respective meanings of science and common sense" Misgeld goes on to write, "while there obviously is a need to clarify the subjective meanings of common sense and science" (p. 196). He then consistently goes on to limit Husserl to the analysis of "subjective meanings." However when I used only the generic term "meaning" without an adjective, it was precisely because I knew it to be a complex phenomenon that had to be differentiated further, as for example, into intersubjective or subjective meaning or cognitive versus emotional meaning, etc.

Now, Misgeld (p. 196) argues that intersubjective meanings are not reducible to subjective meanings. I agree. But'this does not mean that there cannot be a phenomenological analysis of intersubjective meaning. One would have to find out precisely how an individual consciousness could constitute a social meaning as social or how a group of consciousnesses could constitute a social meaning. This is not a special problem for phenomenology because the phenomenological approach to meaning is not at all Cartesian. Meanings transcend the subject. An essence, for example, as a universal invariant meaning transcends the subject that discovers it and it holds universally for all subjects who can apply the methodical procedure. Consequently, it is not at all clear what Misgeld means when he limits Husserl's project to "subjective meanings."

Moreover, Misgeld himself says that common meanings "would have no sense whatsoever were people in the society unable to orient to them through accounting for their meaning subjectively" (p. 196). Husserl says the same thing and even argues that that is why our access to common meanings must be through subjective meanings. But it does not mean that they are identical or that Husserlian analysis is limited to the subjective. It genuinely reaches the social through subjectivity. Here is Husserl:

The historical world, is to be sure, first pregiven as a social-historical world. But it is historical only through the inner historicity of the individuals who are individuals in their inner historicity, together with that of other communalized persons. (1970b, p. 372)

There is no way that Husserlian analysis can be limited to "subjective meanings" in a pejorative sense. Finally, it has often been stated, but apparently bears repeating, that transcendental 
subjectivity is ultimately transcendental intersubjectivity (Husserl, 1970b, p. 185).

\section{(3) Phenomenology and History}

Misgeld states that "any systematic and descriptive examination of common sense and science in all their forms comes too late with respect to our historical situation" (p. 196). He goes on to say that since common sense reasoning is already infiltrated by "scientific or quasi-scientific modes or reasoning" (p. 196) that common sense beliefs are undermined. Thus, he implies: How can one study the relationship between common sense and science when they are already mixed in the lifeworld?

But Misgeld misunderstands the issue here. One does not have to posit "no relationship" between science and common sense in order to analyze them. This seems to imply a purely "content" definition of the two domains. However, in a postscientific age one should not be surprised that common sense contains scientific truisms. Moreover, it should not surprise one that a scientist, outside his professional expertise, may be the embodiment of the common sense of an "educated subculture." Things are indeed complex, but that does not mean they cannot be teased out and analyzed.

Now we have already seen that Husserl's approach to culture was already historical. But to show that Husserl was aware of the complexity of this problem even as he outlined his task, we have only to turn to the following statements:

Making geometry self-evident, then, whether one is clear about this or not, is the disclosure of its historical tradition ... Carried out systematically, such self-evidences result in nothing other and nothing less than the universal a priori of history with all its highly abundant component elements.

We can also say now that history is from the start nothing other than the vital movement of the co-existence and the interweaving of original formations and sedimentations of meaning. (Husserl, 1970b, p. 371)

Note that with this last expression, Husserl implies that history is dynamic (vital movement) involving others (co-existence) and that it is an interplay between original perceptions, intuitions, etc. and prior interpretations of the phenomenon by others. In other words, Husserl recognizes that science, once it appears in history, will encroach upon common sense as formed by other cultural institutions and vice versa.

Moreover, Husserl's appreciation of and allowance for historical factors was not a late development of his. As early as in the Logical Investigations he wrote: "The definitions of a science mirror the stages of that science's development; knowledge of the conceptual 
character of a science's objects, of the boundaries and place of its field, follow the science and progress with it" (Husserl, 1970a, p. 54). Thus, the fact that there are internal developments of a scientific discipline over time is assumed by Husserl.

\section{Phenomenology and Praxis}

Misgeld is impatient with the descriptive analysis of phenomenology and he does not see Husserlian phenomenology as helpful with practical questions. He writes:

As soon as one is aware of the relevant phenomena, one can no longer remain exclusively committed to merely descriptive phenomenology as both Luckmann (building on Schutz) and Giorgi (following Husserl) are. Here I agree with Beekman (1983) and Suransky (1980) with respect to the need for reconsidering phenomenology. One would be compelled to translate insights into practical questions. One will no longer separate research from cultural action and social struggles even if one recognizes that these are not the same. We need to inquire into the point and the possible limits of descriptive accounts of various social situations. (p. 197)

In his challenging of the limits of Husserlian phenomenology, Misgeld also writes:

Thus, hermeneutical and critical theorists can argue (as the orthodox insistence on interminable description in phenomenology cannot) that whenever scientifically directed or technical action and scientific or technical reason are regarded as the only viable form of practical action and of practical reasoning, we are faced with the influence of a technocratic ideology and with scientism as an ideology. (p. 197)

In his view, apparently, only critical theory and/or hermeneutics can address such issues. Unfortunately, Misgeld does not explain why this is the case, except that he seems to imply that Husserlian phenomenology is "merely descriptive."

Once again, for reasons that are not clear, Misgeld arbitrarily delimits the Husserlian project. But since the issue is "praxis" or the ability to deal with "practical questions," let us stick with that. Are phenomenology and praxis antithetical? Not at all! Husserl, after all, developed a method and methods are to be used for concrete analyses. Granted, Husserl did not himself explicitly take on "social issues" in a practical way, but that does not mean that he could not or that he had no interest in them. It is simply a question of how much one can expect from a lifetime of scholarly work. Husserl acknowledged that "infinite tasks" remained. He always wanted a community of scholars working on diverse problems, but this never quite took place to his satisfaction. Nevertheless, since he was conscious of being a founder of a discipline, he preferred to work on theoretical and foundational problems in the hope that if these were set, followers could work out the implications for concrete problems. 
Consequently, I shall sketch part of Husserl's organization of scientific knowledge so that it can be appreciated that he did not think that practical disciplines were impossible nor was he against their development.

At the very beginning of Logical Investigations, Husserl (1970a) distinguished among three types of sciences: theoretical sciences, normative sciences and practical sciences. His reason for alerting the reader to these three types of science is that he wanted to establish logic as a fundamental theoretical science rather than a normative science or a practical science or "technology." Theoretical disciplines are for Husserl the truly foundational ones because their interest is directed to investigating matters that cohere in virtue of the inner laws of the phenomena. Both normative and practical disciplines presuppose such a coherence. A normative discipline commits itself to a fundamental value or norm which then determines the unity of that discipline. Husserl (1970a, p. 86) himself provides the following example:

If, e.g. the production, maintenance, increase and intensification of pleasure counts as our good, we shall ask by what objects, or in what subjective and objective circumstances, pleasure is excited. We shall enquire generally into the necessary and sufficient conditions for the emergence, maintenance, increase, etc. of pleasure. These questions taken as targets for our scientific discipline yield a hedonic: This hedonic is normative ethics in the sense of the hedonists.

Thus, by setting the maximization of pleasure as the basic norm, a standard is set whereby concrete experiences of pleasure can be evaluated.

Husserl then goes on to distinguish a practical discipline or technology which "represents a particular case of a normative discipline which arises when the basic norm consists in achieving a universal practical aim" (Husserl, 1970a, p. 87). Thus if one committed oneself to the value that everyone should be able to experience maximum pleasure as often as possible and proceeded to try to bring this about for everyone, one would have a hedonic technology or practical discipline. For Husserl, both normative and theoretical disciplines could have technologies as off-shoots, without being reduced to them. In addition, Husserl thought that there was a necessary logical hierarchy of these three types of sciences: technologies or practical disciplines presupposed normative disciplines and normative disciplines presupposed theoretical ones. Many attempts have been made in history to collapse and identify these types of discipline, but Husserl has argued against such efforts.

Now, the relevance of these distinctions for the issue at hand is as follows. First, there are phenomenological ways of grounding and practicing theoretical, normative and practical disciplines and 
nonphenomenological ways. Second, if one does seek to work within a phenomenological perspective, then Husserl's hierarchical structure of scientific disciplines should be accepted or else a sound phenomenological critique with counter-evidence should be presented. Thirdly, these distinctions clearly demonstrate that Husserlian phenomenology is, in principle, not against praxis. What is true, is that Husserl as a philosopher and founder of phenomenology was most interested in theoretical issues rather than practical ones and performed almost exclusively concrete theoretical analyses. But his vision of and program for phenomenology allows for much more than he was able to accomplish as an individual, including phenomenological analyses of practical issues. Thus, all Misgeld would have to do is set a valuational aim such as "overcoming of cultural conflict situations for the betterment of humanity" or "practical ways of improving education" and then proceed phenomenologically, and he would have what he is looking for. There may not be a specific example in Husserl's writing for such analyses, but then Husserl always invited followers to join him to help work out solutions to problems he did not have time to work through himself.

Actually, it seems as though Misgeld wants to replace a current technology with another technology (in Husserl's sense of the term) only the newer technology has, for him, a better value system. But, for Husserl, in order for the new technology to be genuinely superior to the technology it is replacing, it would have to be grounded in a normative discipline, and that, in turn, in a theoretical discipline. Unless this is worked through, for Husserl, one could never be sure that the newer technology is truly superior.

While I said above that Husserl mostly performed theoretical concrete analyses, there is an obvious exception that is germane to the issue here and that is Husserl's (1970b) analysis of Galilean science in the Crisis. In that study, Husserl used precisely transcendental phenomenology to uncover what Misgeld (1983, p. 198) calls "collective illusions." The study of the development of Galilean science exposed the reification of science into scientism by resituating it back in the lifeworld from which it had become abstracted so that it could no longer simply be taken for granted. It exposed the sedimentation of meaning which was founding scientism by studying how one's constituting had been constituted. Thus, Husserlian phenomenology would oppose the professionalization of professional practices and the reification of phenomena in question even more radically than Misgeld (1983, pp. 198-200) by pointing beyond the professional knowledge produced by a profession to the constituting through which it is constituted as such knowledge. Such undertakings are intended to guide our social and scientific praxis, to liberate us from the taken-for-granted which result from cultural sedimentation and even the "blinders of praxis." As Husserl (1970b, p. 153) himself wrote: "indeed, ultimately, the actual success of 
transcendental phenomenology-depends upon self-reflective clarity carried to its limits." Lest this be misunderstood, Husserl (1981, p. 300) also said: "In this context, every deeper reflection leads back to the fundamental questions of practical reason, which concern both the individual person and the community." In other words, deeper reflection leads us to the problems of practical reason, and Misgeld (1983, p. 197) himself wants us to be better about "practical deliberations."

\section{The Meaning of Phenomenology for Misgeld}

Finally, while Misgeld has argued strenuously against Husserlian phenomenology, and while he has approved of the approach of hermeneutics in Gadamer's sense and critical social theory in Habermas' sense, he still affirms that phenomenology can be helpful in some sense that he leaves entirely unclear. Thus, he speaks of a " 'hermeneutically enlightened' phenomenology" (p. 199), and "Phenomenological inquiry" (p. 199) and "reflection on professional practice requires the employment of phenomenology" (p. 200) and again he speaks of "a hermeneutically reformulated phenomenological urientation" (p. 200) and so on. Thus, we learn that Misgeld prefers some type of "hermeneutic phenomenology," but he also uses just the single term "phenomenology" positively as many times. Since we have demonstrated above that Misgeld has not interpreted Husserl correctly, the meanings of phenomenology that he accepts and disregards have become ambiguous.

The implication of his critique, however, is that hermeneutics and critical theory have leaped into the regions where transcendental phenomenology cannot go. We cannot go into the details here, but Seebohm (1983) has shown how the new hermeneutics and other recent trends in the human sciences have not really solved the question of ground in the human sciences and he goes on to show how a transcendental phenomenological program in principle could. Even if Seebohm is wrong in certain details, the major point is that a transcendental phenomenological approach is applicable to the issues of social-cultural problems and praxis.

The other implication of Misgeld's delimiting phenomenology to post-Husserlian thought is that Husserl is relevant only for theoretical knowledge, but not for "life" or genuinely existential problems. But Husserl's thought in the Crisis belies this attitude. For example, Husserl (1970b, p. 137) writes:

This by no means implies, however, that the life-world epoch-to which further significant movements belong, as we shall show-means no more for human existence, practically and "existentially," than the vocational epoch of the cobbler, or that it is basically a matter of indifference whether one is a cobbler or a phenomenologist, or, also, whether one is a phenomenologist or a positive scientist. Perhaps it will even become 
manifest that the total phenomenological attitude and the epoch belonging to it are destined in essence to effect, at first, a complete personal transformation, comparable in the beginning to a religious conversion, which then, however, over and above this, bears within itself the significance of the greatest existential transformation which is assigned to mankind as such.

While Husserl himself did not live long enough to fulfill this ambition (but then, who could?) this is no reason to condemn the program as such.

\section{Conclusion}

In this article we have been clarifying the possibilities of applying in principle "pure phenomenology" since Misgeld interpreted Husserlian thought in much too narrow a way. I think it is clear that Husserlian phenomenology, when considered comprehensively and unbiasedly can be applied to the problems of society and the human sciences in highly diverse and fruitful ways. We have shown that Husserlian phenomenology is relevant to culture and to history, that it deals with all types of meanings from the idiographically subjective to the most universal and that Husserl's method allows for many styles of praxis. Because Husserl was comprehensive and preferred to work on foundational and theoretical problems, his relevance for social reality and human problems is often remote rather than proximate, but that does not make him irrelevant. After all, who could put the crisis of our times more eloquently than Husserl?

The spiritual need of our time has, in fact become unbearable. Would that it were only theoretical lack of clarity regarding the sense of the "realiny" investigated in the natural and humanistic sciences that disturbed our peace ... Far more than this, is the most radical vital need that afflicts us, a need that leaves no point of our lives untouched. All life is taking a position, and all taking of position is subject to a must. (1965, p. 140)

\section{Notes}

1. I would like to thank Larry Davidson for helping me to research aspects of this article.

\section{References}

Beekman, T. (1983). Human science as dialogue with children. Phenomenology + Pedagogy, 1(1), 36-44.

Giorgi, A. (1983). Common sense and science: Adversaries or friends? A comment on Luckmann. Phenomenology + Pedagogy, 1, 80-88.

Husserl, E. (1965). Philosophy as rigorious science. In Q. Lauer (Trans. and Ed.), Phenomenology and the crisis of philosophy. New York: Harper and Row. 
Husserl, E. (1970a). Logical investigations (J. N. Findlay, Trans.). New York: Humanities Press. (Original work published in 1900)

Husserl, E. (1970b). The crisis of European sciences and transcendental phenomenology (D. Carr, Trans.). Evanston, IL: Northwestern University Press. (Original work published in 1954)

Husserl, E. (1981). Renewal: Its problem and method. In P. McCormick \& P. Elliston (Eds.), Husserl: Shorter works. Notre Dame, IN: University of Notre Dame Press.

Luckmann, T. (1983). Common sense, science and the specialization of knowledge. Phenomenology + Pedagogy, 1, 59-76.

Misgeld, D. (1983). Phenomenology, social science and the social service professions: The case for the integration of phenomenology, hermeneutics and critical social theory (A reply to Luckmann and Giorgi). Phenomenology + Pedagogy, 1, 195-214.

Seebohm, T. (1983). The new hermeneutics, other trends, and the human sciences from the standpoint of transcendental phenomenology. In H. J. Silverman, J. Sallis, \& T. M. Seebohm (Eds.), Continental philosophy in America. Pittsburgh, PA: Duquesne University Press.

Suransky, V. (1980). Phenomenology as an alternative research paradigm and a force for social change. Madison, WI: University of Wisconsin Press.

Taylor, C. (1971). Interpretation and the sciences of man. Review of metaphysics, 25(3), 1, 32, 35-45. 